\title{
MRI-US fusion guided prostate biopsy: how I do it
}

\author{
Iulia Andras ${ }^{1,2 *}$, Emanuel-Darius Cata ${ }^{1,2 *}$, Dana Crisan ${ }^{3}$, David Andras ${ }^{4}$, Teodora Telecan ${ }^{2}$, \\ Stefana Tartamus², Maximilian Buzoianu', Andrei Maga ${ }^{2}$, Nicolae Crisan ${ }^{1,2}$ \\ * the authors share the first authorship
}

${ }^{1}$ Urology Department, Iuliu Hatieganu University of Medicine and Pharmacy, ${ }^{2}$ Urology Department, Municipal Hospital, ${ }^{3}$ Internal Medicine Department, $5^{\text {th }}$ Medical Clinic, Iuliu Hatieganu University of Medicine and Pharmacy, ${ }^{4}$ General Surgery Department, $1^{\text {st }}$ Surgical Clinic, Iuliu Hatieganu University of Medicine and Pharmacy, Cluj-Napoca, Romania

\begin{abstract}
Multiparametric magnetic resonance imaging (MRI) and MRI-guided prostate biopsy have become the standard for prostate cancer diagnosis. As their implementation is relatively recent, experience is still limited in various centres. MRI-guided biopsy requires basic knowledge in prostate MRI and ultrasound (US), but also in the image processing protocol specific for each device. Standardization of the method is needed to ensure the best results in terms of diagnosis accuracy. We hereby present our technique for MRI-US fusion guided prostate biopsy and the outcomes after performing more than 600 procedures.

Keywords: multiparametric MRI; MRI-US fusion guided biopsy; prostate cancer
\end{abstract}

\section{Introduction}

Multiparametric magnetic resonance imaging (MRI) and MRI-guided prostate biopsy have become an integral part of prostate cancer (PCa) diagnosis. Currently, the European Association of Urology (EAU) Guidelines recommend MRI-guided prostate biopsy both in the initial and repeat biopsy setting [1]. Despite leading to significant improvement in the diagnosis of $\mathrm{PCa}$ [2], their implementation worldwide has been hampered by the increased costs [3]. As such, experience is still limited in various centres [4].

MRI-ultrasound (US) fusion biopsy entails that the ultrasound machine is able to perform a real-time overlay of the MRI and US volumes in order to assist a targeted biopsy. These systems are the most widely used due to

Received 19.01.2020 Accepted 06.03.2021

Med Ultrason

2021, Vol. 23, No 3, 355-360

Corresponding author: Assist. Prof. David Andras, MD

General Surgery Department,

$1^{\text {st }}$ Surgical Clinic, Iuliu Hatieganu University

of Medicine and Pharmacy,

Cluj-Napoca, Romania

8 V. Babes street, 400012, Cluj-Napoca

E-mail: andrasdavid88@gmail.com the best cost/effectiveness ratio [3,5]. MRI-US fusion biopsy requires basic knowledge of prostate MRI and US, together with image processing protocol, which is specific for each device. Prostate MRI is a relatively new addition to the urologic practice and urologists, who are performing the biopsy, are frequently inexperienced in interpretation. Thus, the learning curve of the procedure might be cumbersome [6]. Therefore, minimal training in MRI reading and standardization of the MRI-US fusion technique have the potential to ensure a good diagnostic accuracy.

The aim of the current paper is to present our standardized technique and outcomes of MRI-US fusion guided prostate biopsy. A real-time software-based MRI-US fusion system with rigid registration was employed in all cases.

\section{Prostate MRI}

Prostate multiparametric MRI contains two anatomic sequences ( $\mathrm{T} 1$ and $\mathrm{T} 2$ weighted imaging - WI) and two functional sequences (diffusion weighted imaging - DWI and dynamic contrast enhancement - DCE) [7]. T1WI is mostly used to detect haemorrhage, particularly in patients with prior prostate biopsy. In T2WI, PCa appears as 
a low signal intensity area, which can be lentiform or of irregular shape, located mainly at the peripheral zone of the prostate. High signal intensity on DWI and low signal intensity on derived apparent diffusion coefficient map are indicative for $\mathrm{PCa}$, suggesting increased cellularity and decreased water motion. Furthermore, on DCE-WI, PCa shows early and intense uptake of gadolinium. By combining these sequences, the radiologist gives every suspicious lesion a Prostate Imaging Reporting and Data System (PIRADS) score, aiming to quantify the risk of significant PCa (PIRADS 1: very low - PIRADS 5: very high) [8].

\section{Indications of MRI-guided biopsy}

According to the EAU Guidelines [1], a prostate biopsy is indicated in cases of high prostate specific antigen (PSA), suspicious digital rectal examination or suggestive findings at imaging (MRI). When MRI is available, it should be performed prior to the biopsy. The imaging trigger for prostate biopsy is the presence of a lesion with a PIRADS score equal or higher than 3 . For biopsy naive patients, the combination between targeted and systematic biopsy is strongly recommended, as there is an important added value of both for the diagnosis of clinically significant disease. In patients with prior negative biopsy, systematic cores could potentially be omitted (degree of recommendation according to the EAU Guidelines - weak) [1].

\section{MRI-US fusion biopsy technique}

\section{Preparation of the MRI images}

The minimal requirements for prostate mpMRI are as follows: at least $1.5 \mathrm{~T}$ scan with $\mathrm{T} 2$ weighted imaging (T2WI), diffusion weighted imaging and dynamic contrast enhancement. The protocol of our clinic has been detailed [9]. A written report and drawing, if possible, to describe and illustrate the characteristics of the lesion in terms of number, dimension, location and PIRADS score is required from the radiologist.

For the actual prostate biopsy, axial and sagittal planes of T2WI are used. The first step is to import these two sequences into the US machine. In order to have a 3-dimensional real-time representation of the prostate and the lesion, the two sequences need to be aligned and synchronized. The easiest way to perform this step is in the axial view of the prostate. The two MRI volumes are visible on the screen: the original axial view and the secondary axial view reconstructed from the sagittal images (fig 1A). This secondary view is of lower quality due to the fact that it is reconstructed by the software.

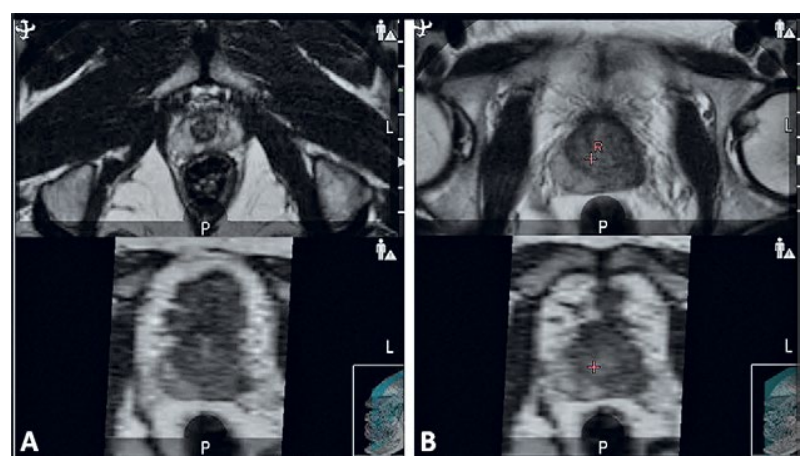

Fig 1. Preparation of the MRI images, axial view in the upper quadrant, axial reconstruction from the sagittal view in the lower quadrant: A. Sequences are not synchronized, B. Synchronization of sagittal and axial volumes of MRI (the red cross marks a small cyst visible on both sections)

It is important to find an anatomical element which is visible on both sequences to be able to perform the synchronization (e.g. prostatic cysts, calcifications) (fig 1B). Both sequences should be positioned in the slice where the common anatomical element of choice can be seen best in order to perform the synchronization. Before synchronization, it is possible to scroll separately in every sequence. After synchronization, the two volumes will scroll together. An isotropic MRI would offer the same quality of images in all planes; thus, this step would not be necessary.

The next step is to perform the contouring of the lesion/s. Based on the radiologist's description or drawing, the lesion is identified by scrolling through the T2WI images. Usually, this step is performed also in the axial view. The US allows only standard shapes for lesion demarcation; thus, it is important to identify and target the centre of the lesion. Biopsy of the peripheral area of a lesion is not always accurate in terms of the Gleason score and sometimes might even miss the tumoral tissue. For lesion contouring, a sphere is most frequently used, with the "+" sign showing the plane of maximal diameter (fig 2A). After contouring the lesion, a verification should be performed in the sagittal view, to confirm that the lesion is accurately delineated in reference with the MRI description (fig 2B).

Due to the fact that the US is based on a rigid registration software, it will not account for the deformation of the prostate secondary to the endorectal US. As such, different markers should be used to confirm that the change in prostate volume is minimal. For example, two straight lines can be used as markers of the anterior and posterior surfaces of the prostate. These markers are inserted in the sagittal view, in the same plane as the maximum diameter of the lesion (fig 3). Thus, if the prostate on the US is not bordered by these markers during the biopsy, the opera- 

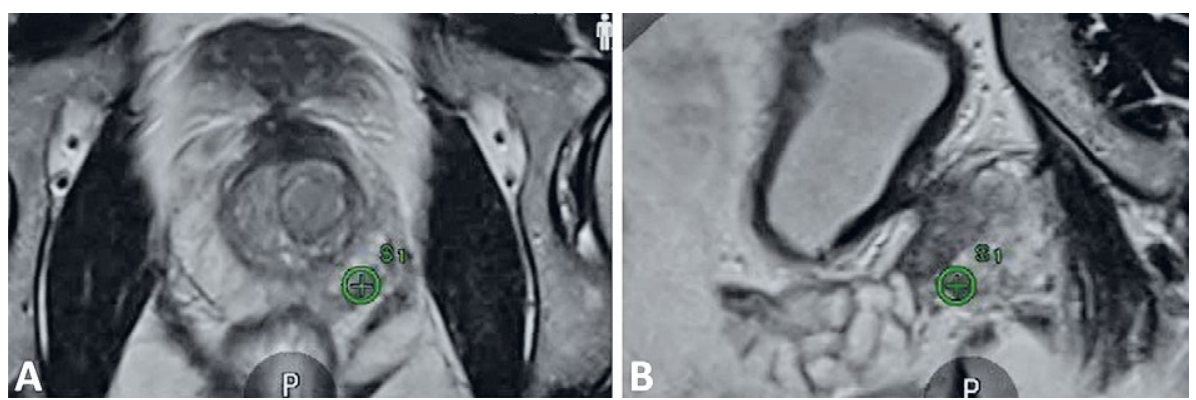

Fig 2. Preparation of the MRI images: A. Contouring of the lesion in axial plane, B. Sagittal plane view to confirm correct delineation of the lesion

tor should adjust the pressure of the endorectal probe in order to ensure a minimal deformation of the prostate. The deformation of the prostate leads to change in the lesion location and negatively affects the accuracy of the targeted biopsy.

The last step before performing the biopsy is to identify an anatomical element which will be used for the synchronization between MRI and US volumes. For this, the urethral axis is most frequently employed. The urethra is marked with a straight line in the sagittal MRI view of the prostate, from the bladder neck until the apex of the gland (fig 4A). In cases with voluminous adenoma and median lobe, the urethra might not be completely visible in the sagittal view. In these cases, we use two markers - the first to approximate the urethral axis and the second to delineate the posterior surface of the median lobe. These two markers should intersect at the apex of the prostate (fig 4B).

All these steps can be performed and memorized prior to the procedure, in order to decrease the time of the biopsy and the potential anxiety of the patient.

\section{Performing the procedure}

The patient is positioned in left lateral decubitus, in a $45^{\circ}$ angle to the long axis of the operating table, with the knees bent on the abdomen. The magnetic field transmitter is positioned in front of the patient, at the level

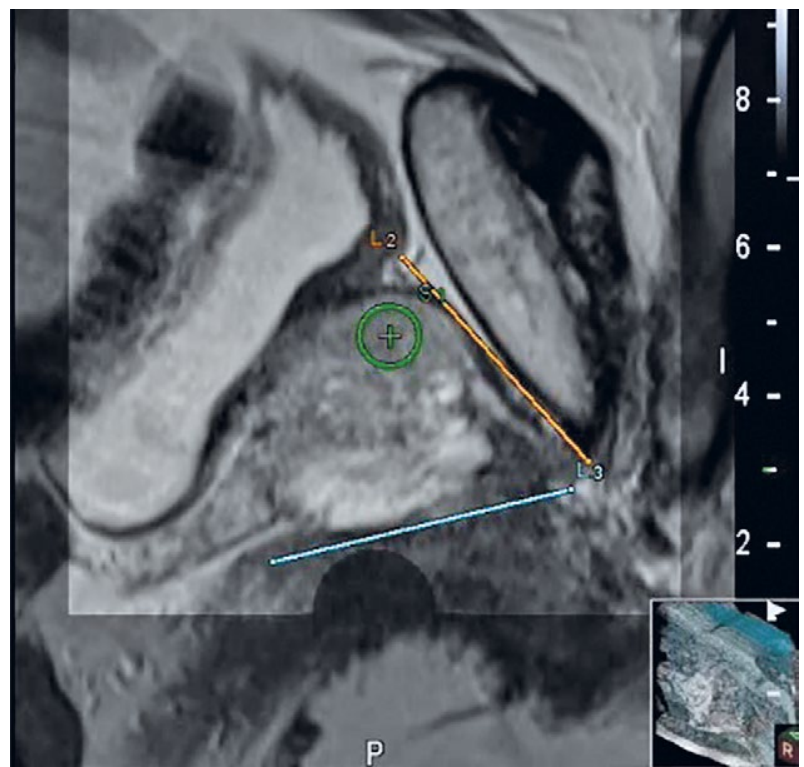

Fig 3. Preparation of the MRI images: marking the anterior (orange line) and posterior (blue line) surface of the prostate to account for potential deformation during the biopsy

of the abdomen. Care is taken that the transmitter is not in direct contact with the patient to avoid its movement during the procedure. Also, attention should be taken to avoid metallic instruments or operating tables with me-
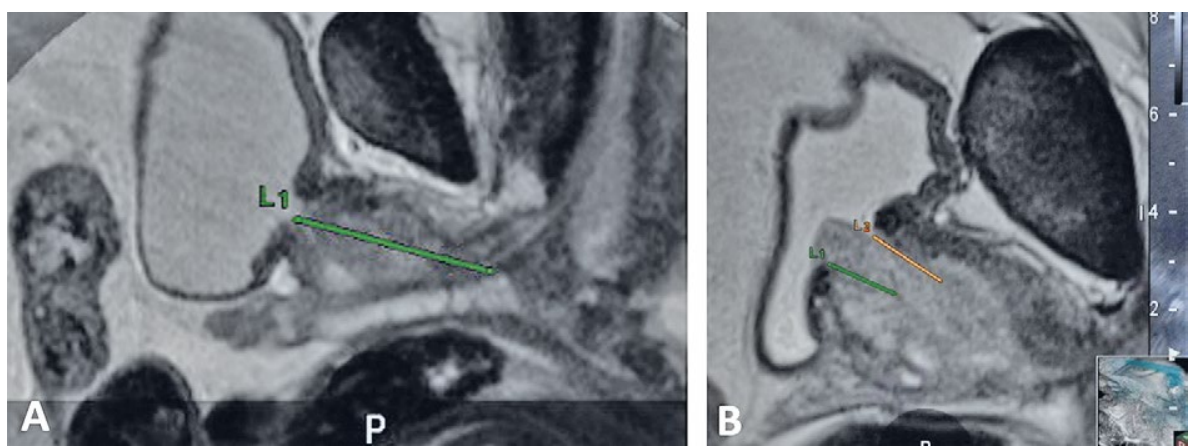

Fig 4. Preparation of the MRI images, marking of the urethra: A. Normal prostate vs B. Median lobe 

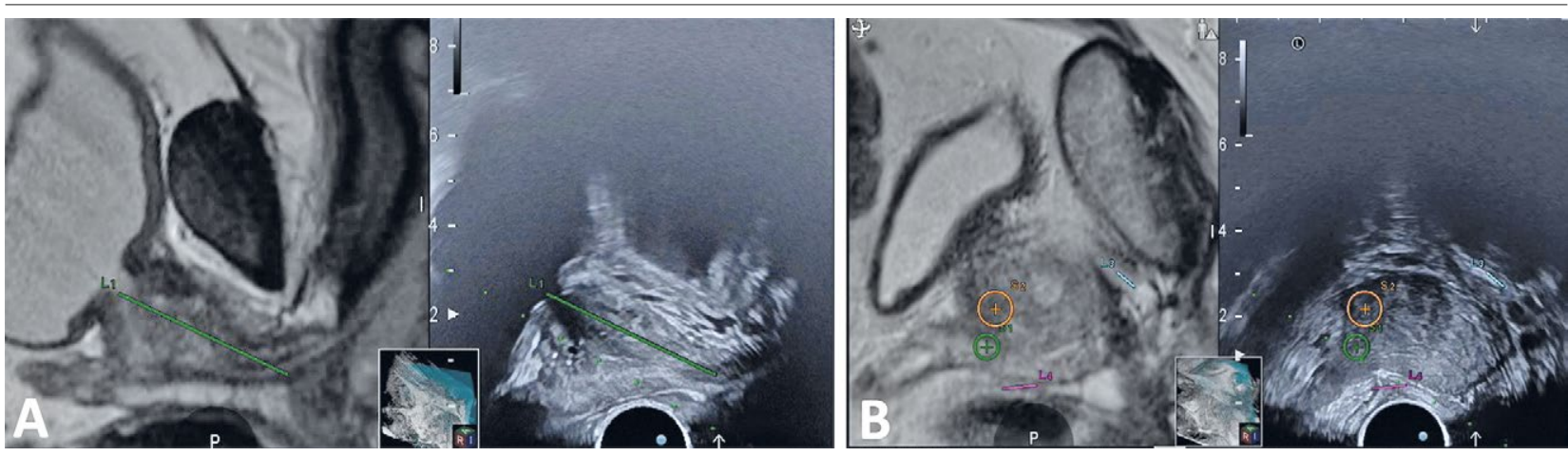

Fig 5. Performing the biopsy: A. MRI-US synchronization based on the urethral axis. B. Lesion visualization for targeted biopsy

tallic edges located very close to the transmitter as they will interfere with the magnetic field. The magnetic field sensor and needle guide are positioned on the endorectal probe as recommended by the producer of the ultrasound machine. Local anaesthesia is performed by endorectal instillation of lidocaine-containing gel. The biopsy is performed by endorectal route.

The procedure begins with an endorectal ultrasound in the sagittal view, aiming to identify the local anatomy. If the urethral axis is the chosen synchronization marker, then the aim is to identify it on the ultrasound and position the marker accordingly. The screen of the ultrasound machine should now be split in two: in the left side, there should be the MRI T2WI in sagittal view in the plane of the urethral axis, whereas in the right side there should be the sagittal view of ultrasound showing the urethral axis. At this moment, synchronization of the two volumes can be performed and real-time fusion of the images will be visible (fig 5A). The US machine will translate all the markers from the MRI unto the ultrasound, and the two volumes will be synchronized with the movement of the endorectal probe, assisting a targeted biopsy. After image registration and before beginning the biopsy, a last check should be performed to assess that the synchronization between the MRI and US volume is correct by scanning the whole prostate and visually comparing the two images. Different anatomical elements visible on both imaging methods (cysts, calcifications) can be used to confirm the registration. If the registration is not suitable and the operator visually assesses that the MRI and US volumes are not similar, registration can be reset and performed again until accurate fusion is achieved. Too much pressure on the prostate secondary to the intrarectal probe can lead to the deformation of the prostate in the transversal axis. Thus, when scanning the prostate in fusion mode the operator will see the lateral part of the prostate on US, whereas on MRI periprostatic tissues will be visible. We recommend performing the checklist illustrated in figure 6 to ensure the accuracy of MRI-US fusion biopsy.

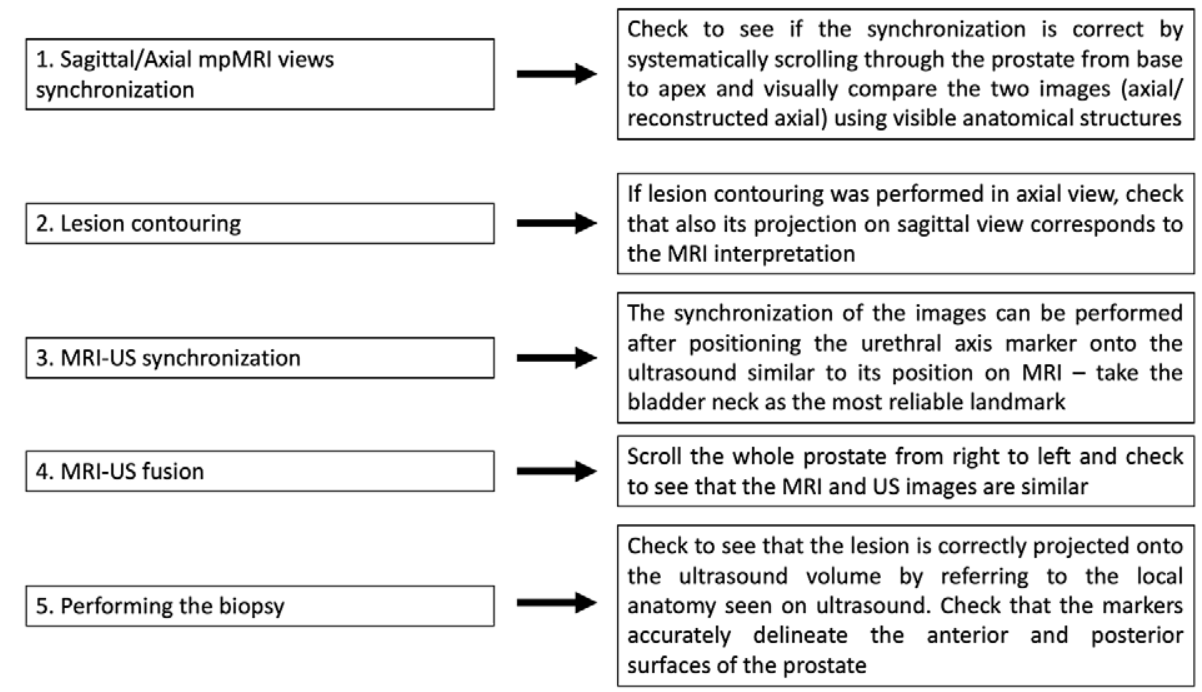

Fig 6. MRI-US fusion biopsy accuracy checklist 
If the checklist has been confirmed and the registration is correct, then targeted biopsy can be performed. The transducer is placed in the plane of maximum dimension of the lesion. A check should be performed that the markers delineating the anterior and posterior surfaces of the prostate are similar on MRI and ultrasound, confirming the minimal deformation of the gland. The biopsy needle should aim for the centre of the lesion, marked by the sign "+". Usually, the MRI-US fusion prostate biopsy comprises targeted cores and systematic sampling. It is recommended to begin with the targeted cores (we currently perform 2-4 per lesion, depending on the dimension) because after the first biopsies, the local oedema might lead to gland deformation and changes in the lesion location. The biopsy needle is advanced until reaching the lesion and then a biopsy is performed (fig 5B). A special situation is when the lesions are located at the anterior fibromuscular stroma, where the needle might potentially injure the Santorini complex. During the biopsy, the needle advances approximately $22 \mathrm{~mm}$; thus, at least this distance should be maintained between the tip of the needle and the anterior surface of the prostate. After performing the targeted biopsy, systematic sampling according to the standard scheme (6 cores/lobe, base/ mid-gland/apex, lateral/medial) is advised. Every biopsy core is sent separately to the pathology department, and its location is registered. The most frequent problematic situations that can be encountered during MRIUS fusion biopsy and their solutions are summarized in Table I.

\section{Our experience}

Between October 2017 and December 2020, a number of 611 MRI-US fusion prostate biopsies were performed in our department. The median age of the patients was 64 years (IQR: 60-69) and the median PSA was 7 $\mathrm{ng} / \mathrm{ml}$ (IQR: $5.08-10.1)$. Almost a quarter (23.4\%) of patients had a history of at least one prostate biopsy. Lesions located in the peripheral area of the prostate were found in $59.8 \%$ of patients, whereas in $40.2 \%$ of cases the lesion was located in the anterior or transitional areas. Overall $\mathrm{PCa}$ and clinically significant $\mathrm{PCa}$ (csPCa, defined as Gleason group $>1$ ) detection rates were $47.38 \%$ and $35.35 \%$. The overall and csPCa detection rate according to the PIRADS score were as follows: PIRADS $3-22.85 \%$ / 10.47\%, PIRADS $4-47.66 \%$ / 38.26\%, PIRADS $5-76.10 \%$ / $62.83 \%$.

\section{Conclusion}

MRI-guided prostate biopsy has recently become the standard for the diagnosis of PCa. As MRI-US fusion systems are most commonly used for guidance, standardization of the technique is necessary to ensure high diagnostic accuracy from the beginning of the learning curve.

Acknowledgement. The study was supported by a grant from the Romanian Ministry of Education and Research, CNCS - UEFISCDI, project no. PN-III-P1-1.1PD-2019-1237, within PNCDI III.

Table I. Troubleshooting during MRI-US fusion biopsy

\begin{tabular}{lll}
\hline What seems to be the problem & The most probable cause & What should you do \\
\hline $\begin{array}{l}\text { No signal received by the mag- } \\
\text { netic field sensor }\end{array}$ & Connection trouble & $\begin{array}{l}\text { Check the connecting cable between magnetic field } \\
\text { transmitter and ultrasound. }\end{array}$ \\
& $\begin{array}{l}\text { Check that the magnetic field generator is turned on. } \\
\text { Position the magnetic field transmitter as close as possible } \\
\text { to the patient abdomen. }\end{array}$
\end{tabular}

After MRI-US fusion, the image Magnetic field interference starts to tremble

When scanning the prostate in fusion mode, the image seems inverted as compared to MRI

After MRI-US fusion, the lesion is projected outside the prostate

During the biopsy, the MRI-US fusion image suddenly changes its position on the screen / disappears

During the biopsy, the needle does not follow the guidance line

\section{Left/Right inversion}

Prostate deformation due to endorectal probe

The patient changed the position of magnetic field transmitter

Incorrect positioning of the biopsy kit on the ultrasound probe
Check if there is any metal (instruments, bed edge) close to the magnetic field transmitter and remove it

Check to see if the transducer is correctly positioned with respect to left/right settings.

Check to see if the magnetic field sensor is correctly positioned on the ultrasound probe.

Adjust the pressure on the endorectal probe to minimize prostate deformation

Reposition the magnetic field transmitter, reset the registration and perform again the fusion of the MRI and US

Check and correct the positioning of the biopsy kit on the ultrasound probe. 


\section{References}

1. Mottet N, van den Bergh RCN, Briers E, et al. EAUEANM-ESTRO-ESUR-SIOG Guidelines on Prostate Cancer-2020 Update. Part 1: screening, diagnosis and local treatment with curative intent. Eur Urol 2021;79:243-262.

2. van der Leest $M$, Cornel $E$, Israël $B$, et al. Head-to-head comparison of transrectal ultrasound-guided prostate biopsy versus multiparametric prostate resonance imaging with subsequent magnetic resonance-guided biopsy in biopsy-naïve men with elevated prostate-specific antigen: a large prospective multicenter clinical study. Eur Urol 2019;75:570-578.

3. Venderink W, Govers TM, De Rooij M, Fütterer JJ, Sedelaar JPM. Cost-effectiveness comparison of imagingguided prostate biopsy techniques: Systematic transrectal ultrasound, direct in-bore MRI, and image fusion. AJR Am J Roentgenol 2017;208:1058-1063.

4. Zhen L, Liu X, Yegang C, et al. Accuracy of multiparametric magnetic resonance imaging for diagnosing prostate
Cancer: A systematic review and meta-analysis. BMC Cancer 2019;19:1244.

5. Tooker GM, Truong H, Pinto PA, Siddiqui MM. National survey of patterns employing targeted MRI/US guided prostate biopsy in the diagnosis and staging of prostate cancer. Curr Urol 2019;12:97-103.

6. Kasabwala K, Patel N, Cricco-Lizza E, et al. The learning curve for magnetic resonance imaging/ultrasound fusionguided prostate biopsy. Eur Urol Oncol 2019;2:135-140.

7. Wu RC, Lebastchi AH, Hadaschik BA, et al. Role of MRI for the detection of prostate cancer. World J Urol 2021;39:637-649.

8. Turkbey B, Rosenkrantz AB, Haider MA, et al. Prostate Imaging Reporting and Data System Version 2.1: 2019 Update of Prostate Imaging Reporting and Data System Version 2. Eur Urol 2019;76:340-351.

9. Andras I, Crisan D, Cata E, et al. MRI-TRUS fusion guided prostate biopsy - initial experience and assessment of the role of contralateral lobe systematic biopsy. Med Ultrason 2019;21:37-44. 